\title{
The metabolic footprint of Clostridia and Erysipelotrichia reveals their role in depleting sugar alcohols in the cecum
}

Connor R. Tiffany' ${ }^{1}$, Jee-Yon Lee ${ }^{1}$, Andrew W. L. Rogers ${ }^{1}$, Erin E. Olsan ${ }^{1,2}$, Pavel Morales ${ }^{3}$, Franziska Faber ${ }^{1,4 \dagger}$ and Andreas J. Bäumler ${ }^{1 *+}$ (I)

\begin{abstract}
Background: The catabolic activity of the microbiota contributes to health by aiding in nutrition, immune education, and niche protection against pathogens. However, the nutrients consumed by common taxa within the gut microbiota remain incompletely understood.

Methods: Here we combined microbiota profiling with an un-targeted metabolomics approach to determine whether depletion of small metabolites in the cecum of mice correlated with the presence of specific bacterial taxa. Causality was investigated by engrafting germ-free or antibiotic-treated mice with complex or defined microbial communities.

Results: We noted that a depletion of Clostridia and Erysipelotrichia from the gut microbiota triggered by antibiotic treatment was associated with an increase in the cecal concentration of sugar acids and sugar alcohols (polyols). Notably, when we inoculated germ-free mice with a defined microbial community of 14 Clostridia and 3 Erysipelotrichia isolates, we observed the inverse, with a marked decrease in the concentrations of sugar acids and polyols in cecal contents. The carbohydrate footprint produced by the defined microbial community was similar to that observed in gnotobiotic mice receiving a cecal microbiota transplant from conventional mice. Supplementation with sorbitol, a polyol used as artificial sweetener, increased cecal sorbitol concentrations in antibiotic-treated mice, which was abrogated after inoculation with a Clostridia isolate able to grow on sorbitol in vitro.
\end{abstract}

Conclusions: We conclude that consumption of sugar alcohols by Clostridia and Erysipelotrichia species depletes these metabolites from the intestinal lumen during homeostasis.

Keywords: Microbiota, Alcoholic sugars, Polyols, FODMAPs, Clostridia, Erysipelotrichia

\section{Introduction}

The gut microbiota plays an important role in the homeostasis and health of its host organism. A large amount of research has focused on how specific constituents of the

\footnotetext{
*Correspondence: ajbaumler@ucdavis.edu

${ }^{\dagger}$ Franziska Faber and Andreas J. Bäumler contributed equally to this work. ${ }^{1}$ Department of Medical Microbiology and Immunology, School of Medicine, University of California, Davis, One Shields Avenue, Davis, CA 95616, USA

Full list of author information is available at the end of the article
}

microbiota interact with the host to influence immune regulation and metabolism [1], but the metabolic capabilities of the gut microbiota remain incompletely understood. Previous research suggests that while phylogeny can be a good predictor of complex metabolic traits requiring large amounts of genes, such as photosynthesis, it is not necessarily a good predictor of simple metabolic traits like carbohydrate utilization [2]. The relationship between phylogeny and metabolic traits in bacteria is complicated further by the widespread occurrence of lateral gene transfer [3], gene 
loss, and convergent evolution [4]. Nonetheless, some metabolic pathways are conserved among phylogenetic groupings within the gut microbiome [5].

For example, members of the Enterobacteriaceae, a family within the phylum Proteobacteria, lack the capabilities to break down complex carbohydrates and instead rely on respiratory catabolism of fermentation products using terminal electron acceptors such as nitrate and oxygen [6, 7]. Many Bacteroides spp., obligate anaerobes within the phylum Bacteroidetes, are primary fermenters of fiber and mucin-derived polysaccharides [8], encoding a large repertoire of genes associated with glycolytic gene clusters [9], while producing much of the short-chain fatty acid propionate found in the murine gut [10]. Prevotella spp. within the phylum Bacteroidetes can also ferment many plant polysaccharides [11] and the abundance of this genus positively correlates with increased fiber intake in humans [12], but unlike Bacteroides, Prevotella spp. do not produce propionate [10]. Bacteria within Clostridia, a class of sporeforming, obligate anaerobes in the phylum Firmicutes, have been linked to the production of the short-chain fatty acid butyrate $[13,14]$. This metabolite fulfills an important role in the gut, acting as a signaling molecule and carbon source for host colonic epithelial cells (colonocytes) [15, 16]. By driving colonocyte metabolism towards high oxygen consumption through mitochondrial beta oxidation, butyrate helps maintain the epithelium in a state of physiologic hypoxia [17], thereby preserving anaerobiosis in the lumen of the large bowel [18]. Clostridia have also been shown to comprise the bulk of diversity within the human gut microbiome [19], further highlighting the importance of this taxon within the ecosystem of the large intestine. While there is increasing information on the metabolic outputs of Clostridia and their function in mediating gut homeostasis, less is known about what nutrients they consume in vivo.

Clostridia were recently divided into two classes, Clostridia and Erysipelotrichia, which form two sister groups within the phylum Firmicutes [20]. In light of this change in nomenclature, previous work on butyrate production by Clostridia also included Erysipelotrichia isolates [21]. Here we combined germ-free mice engrafted with a defined consortium of Clostridia and Erysipelotrichia isolates with an un-targeted metabolomics approach to determine how these taxa alter the metabolite landscape in the cecum.

\section{Results}

\section{Streptomycin treatment shifts the cecal metabolome by increasing sugar alcohols and sugar acids}

To investigate how antibiotic-treatment changes the murine cecal metabolome, mice $(\mathrm{C} 57 \mathrm{BL} / 6 \mathrm{~J})$ were mocktreated or received a single dose of streptomycin intragastrically and cecal contents were collected 3 days later. The soluble fraction of the cecal contents was then analyzed by un-targeted gas chromatography time-of-flight mass spectrometry (metabolite profiling). Principle component analysis of the samples showed a distinct clustering of mice by treatment group, indicating that streptomycin treatment changes the composition of the murine cecal metabolome (Fig. 1a and Supplementary Table 1). Consistent with a previous report [22], differential abundance of metabolites between streptomycin-treated mice and mock-treated mice revealed that the concentration of many compounds annotated as carbohydrates significantly increased with streptomycin treatment, while the concentration of many compounds annotated as lipids, peptides, or organic acids decreased (Fig. 1b, c). Interestingly, most of the carbohydrates that increased with streptomycin treatment were identified as sugar acids (galactonic acid, glyceric acid, lactobionic acid, ribonic acid, saccharic acid, threonic acid, and xylonic acid) or sugar alcohols (1,5-anhydroglucitol, erythritol, galacitonol, lyxitol, myo-inositol, pentitol, sorbitol, threitol, and xylitol) (Fig. 1d and Supplementary Table 2). This increase in the amount of sugar alcohols and sugar acids raised the possibility that streptomycin treatment might deplete microbes that normally consume these nutrients in the cecum.

\section{Streptomycin treatment depletes Clostridia and Erysipelotrichia from the gut microbiota}

To investigate whether changes in the cecal metabolite profile were linked to specific changes in the community composition, we extracted total genomic DNA from contents of the proximal colon, assembled libraries, and then performed 16S rRNA gene amplicon sequencing (microbiota profiling). Weighted unifrac analysis of the samples showed distinct clustering by treatment group (Fig. 2a), and a significant difference in weighted unifrac distance was observed between streptomycintreated and mock-treated mice (Fig. 2b), indicating that the community structure was different between groups. Clostridia and Erysipelotrichia were the only taxonomic groups to both contain significantly decreased amplicon sequence variants (ASVs) in streptomycin-treated mice relative to mock-treated mice according to negative binomial regression analysis, explaining the difference between the gut microbiota of streptomycin-treated mice and mock-treated mice according to linear discriminant analysis (Fig. 2c). Conversely, Gammaproteobacteria and Bacilli were significantly enriched in streptomycintreated mice. Consistent with previous reports $[18,23$, 24], we observed a decrease in the relative abundance of Clostridia in streptomycin-treated mice (Fig. 2d). Within the Erysipelotrichia, three taxa dropped below the limit of detection in the streptomycin-treated group, whereas 


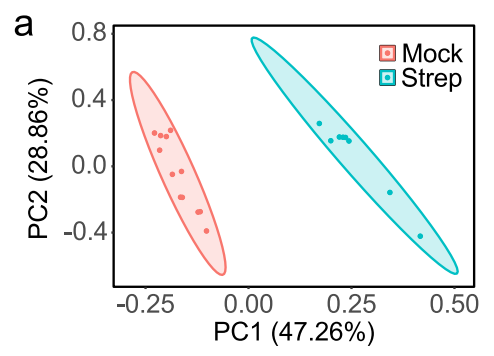

C $\circ$ Carbohydrates $\circ$ Lipids $\circ$ Organic acids $\circ$ Peptides $d$

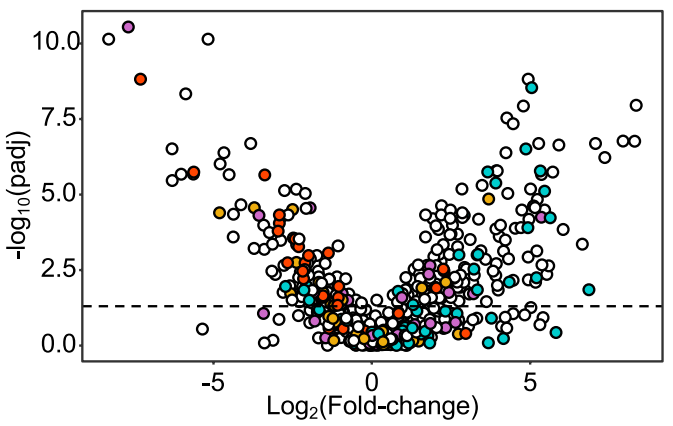

b
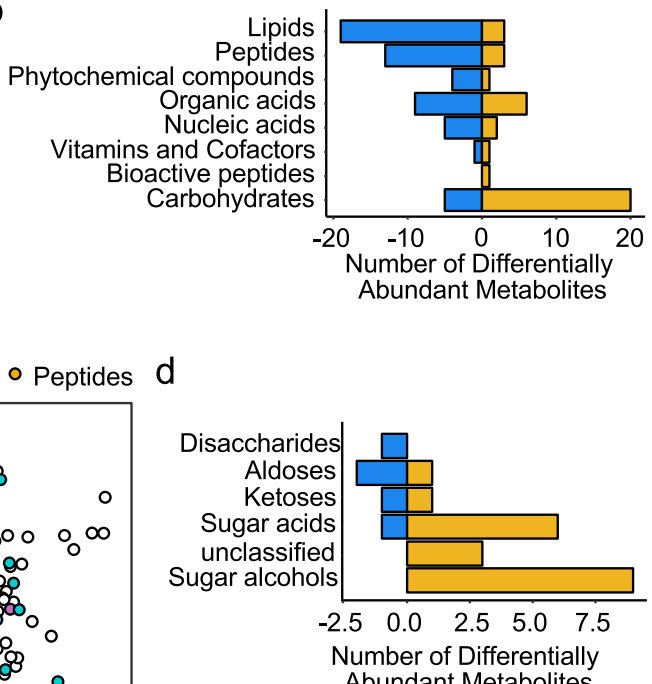

Abundant Metabolites

Fig. 1 Streptomycin treatment alters the mouse cecal metabolome, characterized by an increase in carbohydrates. Mice were mock-treated $(n=12)$ or received a single dose of streptomycin $(n=8)$ and the cecal metabolome was analyzed 3 days later (mice of each group were housed in three different cages each). a PCA plot showing variation of the mouse cecal metabolome by treatment group. $\mathbf{b}$ Bar plot displaying the number of significantly increased and decreased metabolites by metabolite class in streptomycin-treated mice relative to mock-treated mice (FDR corrected $P$ value $=<0.05)$. c Volcano plot of metabolites colored by class. Metabolites with a positive fold-change value increased with streptomycin treatment while metabolites with a negative-fold change value decreased with streptomycin treatment. The dashed line is set at an FDR corrected $P$ value of 0.05. d Bar plot displaying the number of differentially significantly increased and decreased carbohydrates by metabolite subclass in streptomycin-treated mice relative to mock-treated mice (FDR corrected $P$ value $\leq 0.05$ )

the most abundant taxon significantly increased (Fig. 2e and Fig. S1), resulting in an elevated overall relative abundance of Erysipelotrichia in streptomycin-treated mice (Fig. 2d). Differential abundance analysis revealed that the class Clostridia had the most significantly different amplicon sequence variants (ASVs), with most of these decreasing after streptomycin treatment (Fig. 2e). Whereas Clostridia were not the most abundant taxon, this class comprised approximately two thirds of the ASV diversity within the microbiota of mock-treated mice (Fig. 2f). Thus, streptomycin treatment altered the community structure by depleting Clostridia and Erysipelotrichia (Fig. 2c-e), with the former being the taxonomically most diverse class within the gut microbiota (Fig. 2f).

\section{Clostridia and Erysipelotrichia deplete sugar alcohols and sugar acids}

Having established a correlation between Clostridia and Erysipelotrichia depletion and an increase in sugar alcohols and sugar acids, we next wanted to determine whether Clostridia and Erysipelotrichia caused this shift in the cecal metabolome in the absence of potential confounding factors, such antibiotic treatment. To that end, we inoculated germ-free Swiss Webster mice with a consortium originally described as 17 commensal Clostridia isolated from a healthy volunteer [21]. According to current nomenclature, this consortium consists of fourteen Clostridia isolates and three Erysipelotrichia isolates [25] (Fig. 3a and Supplementary Table 3). Five days after colonization, samples were collected and analyzed using microbiota and metabolite profiling. Microbiota profiling revealed that ten Clostridia isolates and three Erysipelotrichia isolates engrafted at levels detectable by $16 \mathrm{~S}$ ribosomal RNA gene amplicon sequencing (Fig. 3b). Principle component analysis showed a distinct clustering pattern between mock-treated germ-free mice and those given the consortium of commensal Clostridia and Erysipelotrichia isolates (Fig. 4a). Remarkably, association of germ-free mice with the commensal consortium (Fig. 4b, c and Supplementary Table 4) produced an equivalent but inverse phenotype to that observed after depleting Clostridia and Erysipelotrichia by treatment with streptomycin (Fig. 1b, c Supplementary Table 1). All significantly different metabolites identified as carbohydrates decreased when germ-free mice were associated with a consortium of commensal Clostridia and Erysipelotrichia isolates (Fig. 4b, c and Supplementary Table 4), which was accompanied by a concomitant increase in a 

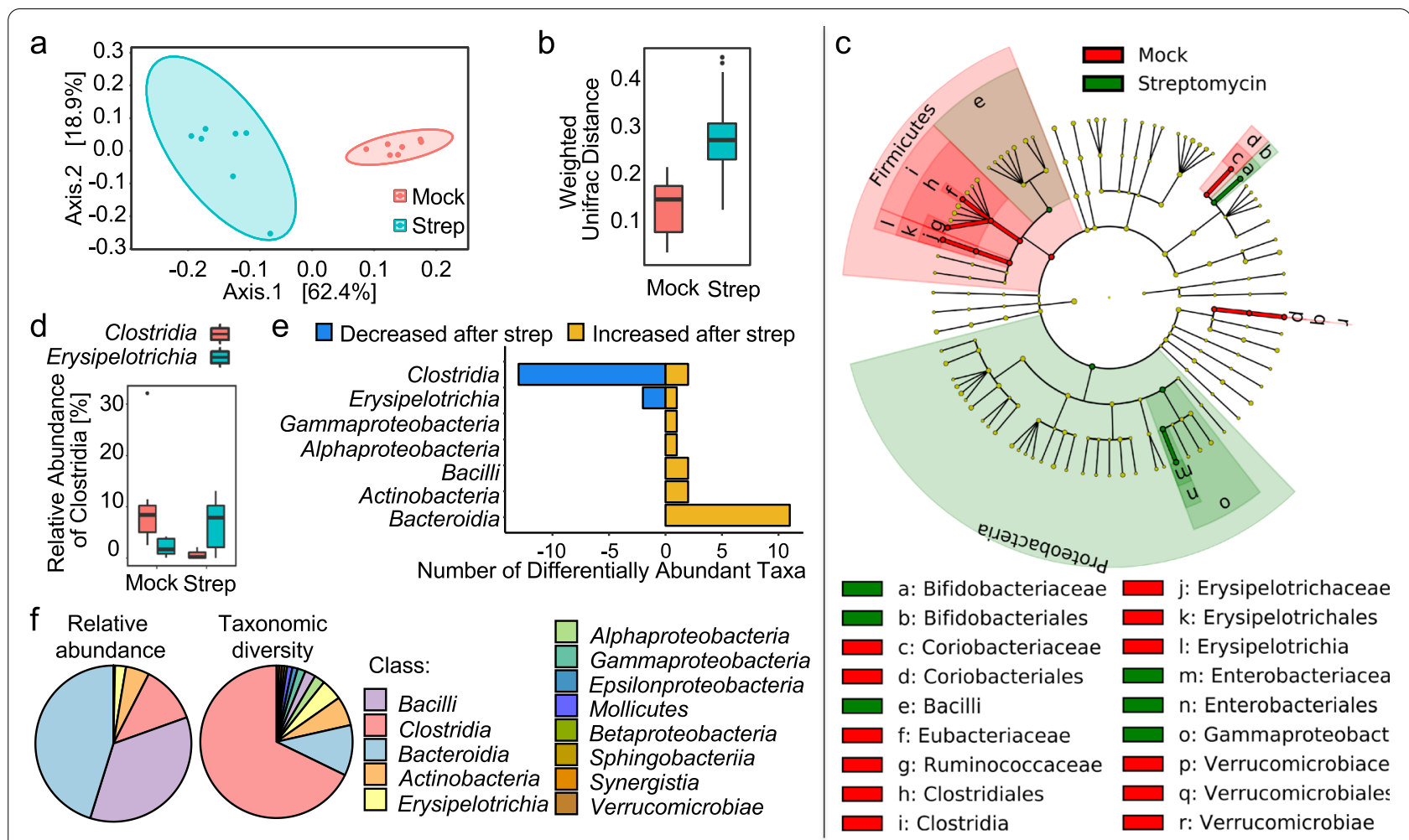

Fig. 2 Streptomycin treatment of mice induces a shift in community structure of the large bowel microbiome. Mice were mock-treated $(n=8)$ or received a single dose of streptomycin $(n=8)$ and bacterial communities in the colon contents were analyzed 3 days later. a Principal coordinate analysis of $16 \mathrm{~S}$ rRNA gene amplicon sequencing of colon contents from mice. $\mathbf{b}$ Box plot of weighted unifrac distance between mock and streptomycin treated mice ( $P$ value $=0.002$ ). c LEFSE cladogram displaying taxa enriched in mock-treated mice (red) or in streptomycin-treated mice (green). Taxa enriched within higher level taxa are indicated by a darker shading. $\mathbf{d}$ Box plots showing the relative abundance of Clostridia and Erysipelotrichia in mock-treated and streptomycin-treated mice. e Significantly increased and decreased amplicon sequence variants by class in streptomycin-treated mice relative to mock-treated mice (FDR corrected $P$ value $\leq 0.05$ ). $\mathbf{f}$ Relative abundance (left pie chart) and taxonomic diversity of ASVs (right pie chart) in mock-treated mice at the class level

plurality of significantly different lipids, peptides, and organic acids. Within carbohydrates, sugar alcohols (erythritol, galactinol, lyxitol, myo-inositol, pentitol, sorbitol, ribitol, and xylitol) and sugar acids (digalacturonic acid, galactonic acid, galacturonic acid, glyceric acid, threonic acid, and xylonic acid) contained the largest number of significantly decreased compounds (Fig. $4 \mathrm{~d}$ and Supplementary Table 5).

Comparing metabolite profiling data from streptomycin-treated mice with those from gnotobiotic mice engrafted with commensal Clostridia and Erysipelotrichia isolates is complicated by the fact that conventional and gnotobiotic mice differed in their genotype (C57BL/6 J versus Swiss Webster) and the composition of their chow. To compare metabolite profiles in the absence of these confounding variables, germ-free Swiss Webster mice received a cecal microbiota transplant from conventionally housed C57BL/6 J mice. Five days after engraftment, cecal samples were collected and analyzed using metabolite profiling. Principle component analysis revealed clustering of samples from mice receiving a cecal microbiota transplant from conventional (C57BL/6) mice with those from mice engrafted with the consortium of commensal Clostridia and Erysipelotrichia isolates (Fig. 4a). Notably, only four carbohydrates (including the alcoholic sugars hexitol, pentitol, and threitol) were depleted in germ-free mice receiving a cecal microbiota transplant from conventional (C57BL/6) mice compared to germ-free mice engrafted consortium of commensal Clostridia and Erysipelotrichia isolates (Fig. 4e, f and Supplementary Table 4). These data suggested that the carbohydrate footprint of a defined microbial community of human Clostridia and Erysipelotrichia isolates was similar to that produced by a complex mouse microbiota.

\section{Polyol utilization by Clostridia and Erysipelotrichia species in vitro and in vivo}

This result demonstrated that a consortium of commensal Clostridia and Erysipelotrichia isolates depletes sugar alcohols and sugar acids in the cecum, but did not 


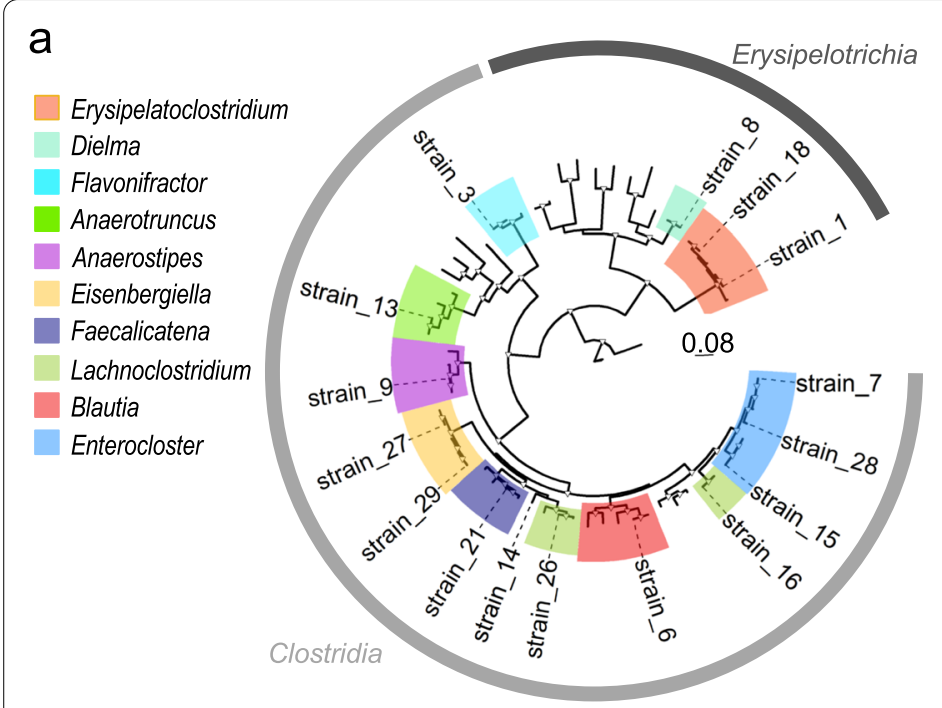

b

Fig. 3 Engraftment of germ-free mice with a defined community of human Clostridia and Erysipelotrichia isolates. a Placement of the members of a defined microbial community (strains $1,3,6,7,8,9,13,14,15,16,18,21,26,27,28$, and 29) on a maximum likelihood phylogeny built from the alignments of 11 total single copy 305 and 50 S ribosomal proteins, which were concatenated together for a total alignment length of 1822 amino acids. The tree encompasses 66 taxa, three of which being Alicyclobacillus species belonging to the class Bacilli that were included as an outgroup. Strain 4 from the defined microbial community was excluded from analysis because NCBI flagged its deposited genomic sequence as chimeric, containing sequences from two strains belonging to the same species, Hungatella hathewayi. Sequences that did not belong to strains within the defined microbial community were obtained from [26]. 150 non-parametric bootstraps were performed to determine confidence of branch placements (white triangle at a node indicate above 70\% confidence). b Germ-free mice were engrafted with a community of 14 Clostridia and 3 Erysipelotrichia isolates and microbiota profiling performed on colon contents obtained five days later

provide direct evidence that these bacteria could utilize these carbon sources for growth. Polyols, such as sorbitol, mannitol, or xylitol, are of particular interest, because they are used as artificial sweeteners in a broad range of processed food products. Thus, we performed an in vitro growth assay using no carbon defined media (NCDM) supplemented with D-gluconic, glucose, or one of the following sugar alcohols: xylitol, D-sorbitol, D-mannitol, myo-inositol, and meso-erythritol. Consumption of various combinations of sugar alcohols was observed for six Clostridia and one Erysipelotrichia isolate, with all seven isolates being able to grow on sorbitol as a carbon source (Fig. 5a).

Sorbitol is commonly used as a supplement to sweeten food. We thus wanted to determine whether consumption of polyols by Clostridia could be observed in streptomycin-treated mice receiving dietary sorbitol supplementation. To this end, mice were maintained on a defined diet containing low concentrations of FODMAPs (Teklad Custom Diet TD.110675, Envigo). Mice were then mock-treated or treated with streptomycin and received $5 \%$ sorbitol in their drinking water. Absolute sorbitol concentrations determined by a colorimetric assay were close to the limit of detection in cecal contents of mock-treated mice. Sorbitol supplementation did not increase the concentration of sorbitol in cecal contents of mock-treated mice, suggesting that an intact microbiota can deplete this dietary supplement (Fig. 5b). In contrast, disruption of the microbiota by streptomycin treatment resulted in a marked increase in cecal sorbitol levels in mice receiving sorbitol supplementation. To investigate whether consumption of sorbitol by microbes was responsible for depleting this dietary supplement, the experiment was repeated with streptomycin-treated mice that were mock-inoculated or engrafted with Anaerostipes caccae (strain 9 from the defined microbial community, originally classified as Clostridium indolis) (Fig. 3a and Supplementary Table 3), which was able to grow in vitro using sorbitol as a carbon source (Fig. 5a). Notably, engraftment of streptomycin-treated mice with A. caccae resulted in a marked depletion of sorbitol (Fig. 5c), thus further supporting the idea that consumption of polyols by Clostridia results in a depletion of these metabolites from the metabolome in the murine cecum.

\section{Discussion}

Members of the class Clostridia perform critical functions within the mammalian gut, including maintenance of hypoxia within the colon [18], providing colonization resistance to enteric pathogens [23, 27], and contributing 


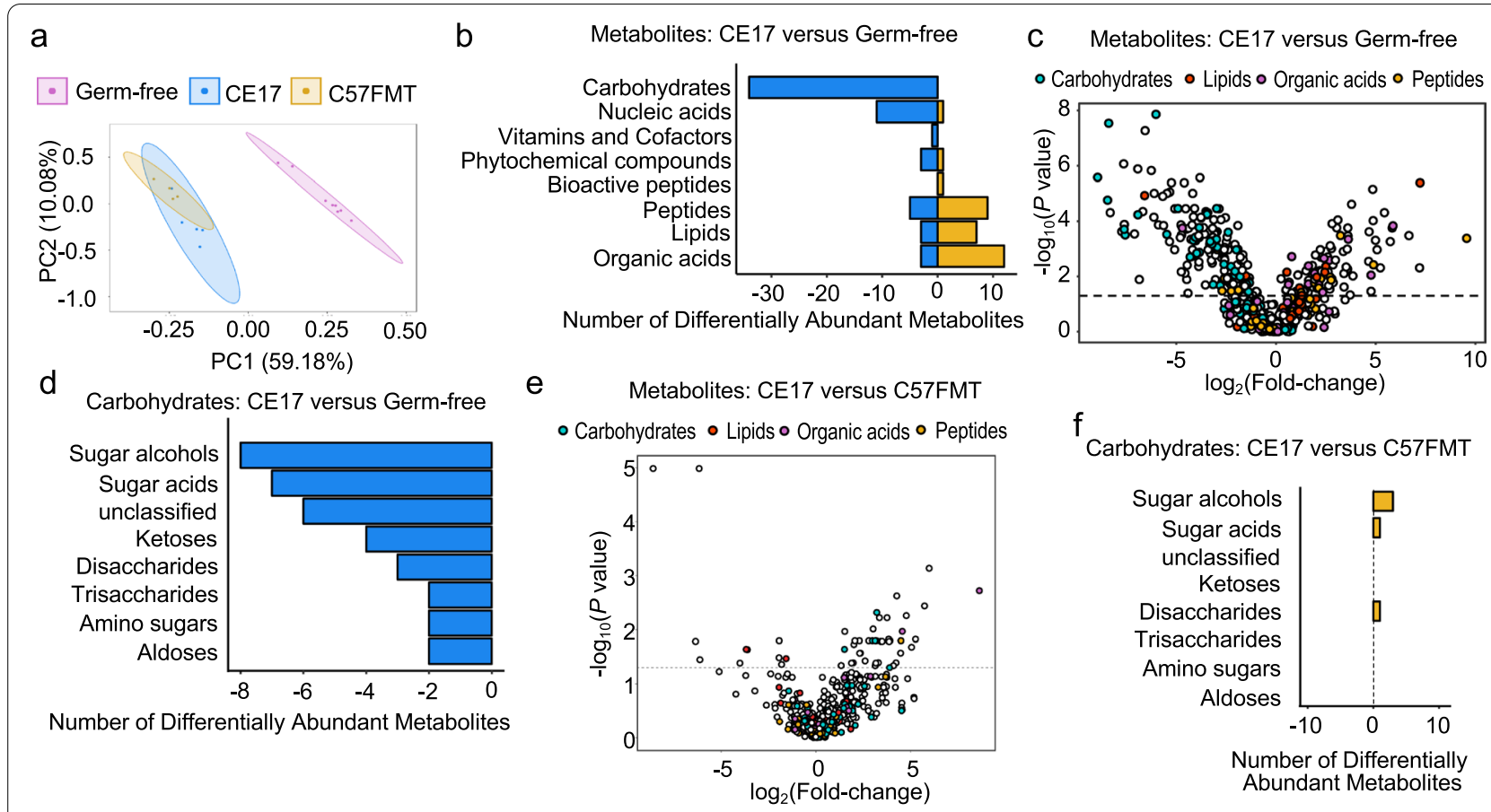

Fig. 4 Colonization of germ-free mice with Clostridia induces a shift in the cecal metabolome. Comparative analysis of the cecal metabolome of germ-free mice $(n=6)$, gnotobiotic mice associated with a defined microbial community containing 14 human Clostridia and 3 human Erysipelotrichia isolates (CE17) ( $n=6$ ), and gnotobiotic mice receiving a cecal microbiota transplant from conventional C57BL/6 J mice (C57FMT) $(n=4)$. a Principle component analysis of the cecal metabolome in the indicated groups of mice. $\mathbf{b}$ Bar plot of significantly increased and decreased metabolites by metabolite class in gnotobiotic mice associated with a defined microbial community (CE17) relative to germ-free mice (FDR corrected $P$ value $\leq 0.05$ ). c Volcano plot of metabolites colored by class. Metabolites with a positive fold-change value increased in mice engrafted with a defined microbial community compared to germ-free mice, while metabolites with a negative fold-change value decreased. The dashed line is set at an FDR corrected P value of 0.05 . d Bar plot of significantly increased and decreased carbohydrates by metabolite subclass in gnotobiotic mice associated with a defined microbial community (CE17) relative to germ-free mice (FDR corrected $P$ value $\leq 0.05$ ). e Volcano plot of metabolites colored by class. Metabolites with a positive fold-change value increased in mice engrafted with a defined microbial community compared to gnotobiotic mice receiving a cecal microbiota transplant from conventional C57BL/6 J mice (C57FMT), while metabolites with a negative fold-change value decreased. $\mathbf{f}$ Bar plot of significantly increased and decreased metabolites by metabolite class in gnotobiotic mice associated with a defined microbial community (CE17) relative to gnotobiotic mice receiving a cecal microbiota transplant from conventional C57BL/6 J mice (C57FMT) (FDR corrected $P$ value $\leq 0.05)$

to the immune education of the mammalian host organism [28]. It is known that Clostridia and their sister group, the Erysipelotrichia, are producers of short-chain fatty acids in the gut, including butyrate [13, 14]. We observed a significant decrease in most organic acids in the gut metabolome following streptomycin treatment, and an increase when Clostridia and Erysipelotrichia were given to germ-free mice. It is thus possible that these organic acids represent Clostridia- and Erysipelotrichia-derived fermentation products, but the un-targeted metabolomics approach performed in this study was unable to detect short-chain fatty acids, because of poor retention and separation of these metabolites in the columns used. We thus focused our analysis on metabolites depleted by Clostridia and Erysipelotrichia, which was indicative of nutrients consumed by members of these taxa. Notably, our data suggested that when commensal Clostridia and Erysipelotrichia were depleted in the murine large bowel, most of the detected sugar alcohols and sugar acids significantly increased, whereas when germ-free mice were associated with a defined community of 14 human Clostridia and 3 human Erysipelotrichia isolates, the inverse held true. Furthermore, Clostridia and Erysipelotrichia isolates (i.e., strains 1, 3, 7, 9, and 29) were able to grow on sugar alcohols as sole carbon sources in vitro and had a combined relative abundance of approximately $12 \%$ within the defined community of 14 human Clostridia and 3 human Erysipelotrichia isolates, thus explaining their ability to deplete polyols in gnotobiotic mice.

Sugar alcohols, such as sorbitol, xylitol, or mannitol, are of interest because of their use as low calorie sweeteners in foods [29]. They belong to a class of sugars known as polyols, which fall under the umbrella of fermentable dietary oligosaccharides, disaccharides, monosaccharides, and polyols (FODMAPs) that are poorly absorbed in the small intestine [30]. As humans lack active 


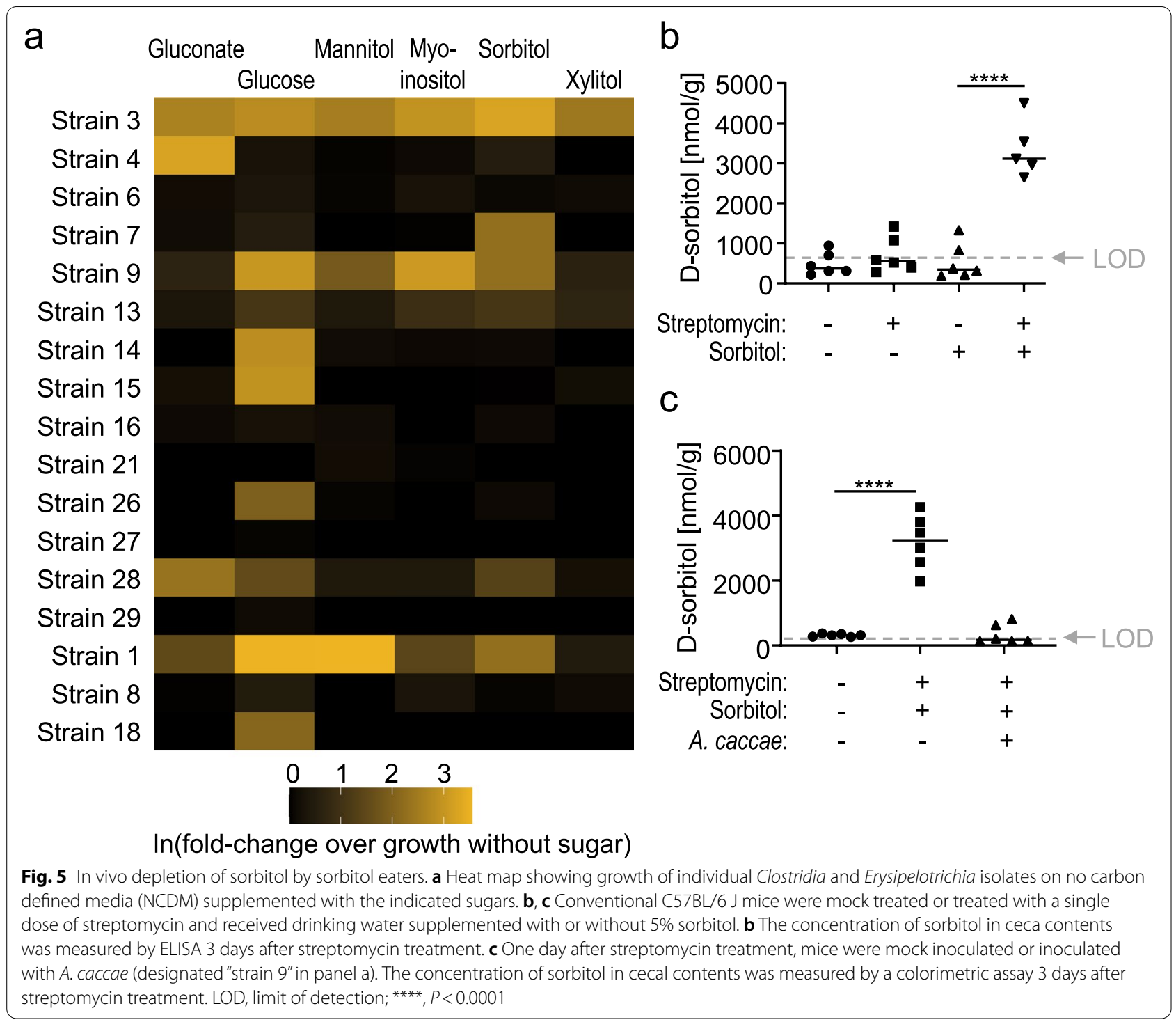

transporters for sugar alcohols in the small intestine, transport relies on passive diffusion, which is why these nutrients are poorly absorbed in the small intestine [30]. As a result, the bulk of dietary sugar alcohols reaches the colon, where they are fermented by the microbiota into short-chain fatty acids [31]. Sugar alcohols are naturally occurring in many fruits and vegetables, making them a prevalent constituent of the human diet [32], but excessive consumption is associated with transient bloating or diarrhea [33]. Excessive consumption of sugar alcohols also is associated with enhanced gas production and diarrhea in patients with irritable bowel syndrome [34, 35] and a diet low in FODMAPs alleviates symptoms in these patients [36]. Our results show that a Clostridia species, A. caccae, that was able to grow on sorbitol in vitro, was able to deplete this polyol when it was provided to antibiotic-treated mice as a dietary supplement.

Interestingly, a previous study suggests that sugar alcohols could be a growth factor for opportunistic enteric pathogens. Clostridioides difficile $(C$. difficile) is an opportunistic pathogen within the class Clostridia, which causes pseudomembranous colitis in individuals undergoing antibiotic therapy [37]. The metabolome of mice treated with the broad-spectrum antibiotic cefoperazone contains a higher abundance of sugar alcohols relative to the metabolome of mock treated animals, similar to what we observed after streptomycin treatment, and this treatment renders mice susceptible to $C$. difficile infection [38]. Additionally, the sugar alcohols sorbitol and mannitol are sufficient for $C$. difficile growth in NCDM in vitro 
[38] and increase toxin production [39]. Furthermore, depletion of alcoholic sugars by the gut microbiota contributes to colonization resistance against $C$. difficile [40]. Similarly, colonization resistance against opportunistic Escherichia coli infection is lost when mice receive supplementation with galacitol, a sugar alcohol [41]. Thus, depletion of sugar alcohols by endogenous Clostridia and Erysipelotrichia species might contribute to colonization resistance against opportunistic enteric pathogens.

\section{Conclusions}

Our results identify members of the classes Clostridia and Erysipelotrichia as the main taxa in the gut microbiota that consume dietary FODMAPs during homeostasis. This finding points to Clostridia and Erysipelotrichia as a possible cause of transient bloating observed after excessive consumption of FODMAPs.

\section{Materials and methods}

Bacterial strains and culture techniques

A community of 14 human Clostridia isolates and 3 human Erysipelotrichia isolates was kindly provided by K Honda [21, 42], and bacteria were cultured as described previously [25].

\section{Animal experiments}

Female C57BL/6 mice, aged 8 weeks, were obtained from The Jackson Laboratory (C57BL/6 J mice) and maintained on gamma irradiated Teklad global $18 \%$ protein rodent diet 2918 (Envigo). C57BL/6 mice were treated with $20 \mathrm{mg} / \mathrm{animal}$ of streptomycin via oral gavage or mock treated and sacrificed 3 days post treatment, and cecal contents were collected, freeze-dried, flash frozen in liquid nitrogen, and stored at $-80{ }^{\circ} \mathrm{C}$ until further processing for metabolomics. Proximal colon contents were collected, snap frozen, and stored at $-80^{\circ} \mathrm{C}$ until further processing for DNA isolation and sequencing.

\section{Sorbitol supplementation}

C57BL/6 mice were maintained on gamma irradiated Teklad Custom Diet TD.110675 (Envigo) and treated with $20 \mathrm{mg} /$ animal of streptomycin via oral gavage or mock treated. One day later, mice received drinking water supplemented with $5 \%$ sorbitol or no supplementation and were inoculated with $200 \mu \mathrm{l}$ of a Clostridia culture by oral gavage. Cecal contents were collected 3 days after streptomycin treatment.

\section{Microbiota transfer}

Male and female germ-free Swiss-Webster mice were bred in house using gamma-irradiated chow (Purina Diet 5066; Charles River) and used for the experiment at 8 weeks of age. For transfer of human Clostridia and
Erysipleotrichia strains into germ-free mice, each strain was individually inoculated into Egerth Gagnon medium and incubated for $48 \mathrm{~h}$. A mixture of equal volumes for each strain was prepared, and each animal was given $200 \mu \mathrm{l}$ of inoculum via oral gavage [25]. For microbiota transfer from conventional C57BL/6 J mice, the entire cecal content of a mouse was collected into $5 \mathrm{~mL}$ pre-reduced sterile PBS inside an anaerobic chamber, homogenized by vortexing and set down for $10 \mathrm{~min}$ to let particles settle. Immediately after, $200 \mu \mathrm{l}$ of the homogenate supernatant was used to inoculate germ-free SwissWebster mice by oral gavage. For the duration of the experiment, mice were kept in sterile cages inside a biological safety cabinet. The mice were sacrificed 5 days after inoculation, and cecal contents were collected, freeze-dried, flash frozen in liquid nitrogen, then stored at $-80{ }^{\circ} \mathrm{C}$ until further processing for metabolomics; proximal colon content was collected, flash frozen in liquid nitrogen, then stored at $-80^{\circ} \mathrm{C}$ until further processing for DNA isolation and sequencing.

\section{In vitro growth assay of Clostridia and Erysipleotrichia isolates}

To assess the ability of the Clostridia and Erysipleotrichia consortium to grow on various individual carbohydrates, we used no carbon defined media (NCDM), which is a modified form of no carbon minimal media (NCMM) [38], but NCDM utilizes below vitamins and amino acids at the following concentrations: thiamine, pantothenate, nicotinic acid, riboflavin, pyridoxine, $\mathrm{p}$-aminobenzoic acid, folic acid, biotin, and vitamin B12 were added in the form of ATCC Vitamin Supplement (ATCC, Manassas, Virginia) to a final concentration of $5 \%(\mathrm{v} / \mathrm{v})$. Calcium, magnesium, manganese, iron, and cobalt salts were added in the form of ATCC Trace Mineral Supplement (ATCC, Manassas, Virginia) to a final concentration of $2 \%(\mathrm{v} / \mathrm{v})$. Indicated carbohydrates were used at a final concentration of $0.5 \%(\mathrm{w} / \mathrm{v})$. We further modified NCDM to accommodate slower growing strains by using Bacto Casamino acids (ThermoFisher) at a concentration of $4.575 \mathrm{~g} / \mathrm{L}$, supplemented with cysteine (400 mg/L), methionine $(27 \mathrm{mg} / \mathrm{L})$, alanine $(72 \mathrm{mg} / \mathrm{L})$, and tryptophan $(30 \mathrm{mg} / \mathrm{L})$. Vitamin $\mathrm{K} 2$ was added at a concentration of $200 \mu \mathrm{L} / \mathrm{L}$. Individual strains were grown anaerobically in a volume of $2 \mathrm{~mL}$ of above medium containing glucose for $24 \mathrm{~h}$. The entire culture volume was then spun down at 12,500 rpm for $3 \mathrm{~min}$, the supernatant was discarded, and the bacterial pellet was resuspended in $750 \mu \mathrm{L}$ of reduced PBS. Twenty-five microliters of the resuspended pellet of each strain was then used to inoculate 2-mL volumes of NCDM containing D-gluconic acid, glucose, myo-inositol, D-mannitol, D-sorbitol, xylitol, or no added carbohydrates in quadruplicate. After $72 \mathrm{~h}$ of 
incubation at $37^{\circ} \mathrm{C}$, growth was assessed by removing the cultures from the anaerobic chamber and reading the optical density at $600 \mathrm{~nm}$. Un-inoculated NCDM was used as a blank reference.

\section{S rRNA gene amplicon sequencing sample preparation, library preparation, and sequencing}

Nucleic acid extraction was done using the DNeasy Blood \& Tissue Kits from Qiagen. DNA concentration was recorded using a Qubit a 2.0 fluorometer. Samples were normalized to $20 \mathrm{ng} / \mu \mathrm{L}$ before library preparation. The $16 \mathrm{sV} 4$ Region was amplified with the $515 \mathrm{f}-806 \mathrm{R}$ primer pair. Each $10 \mu \mathrm{L}$ amplicon-PCR reaction consisted of 7 $\mu \mathrm{L}$ of TailorMix 2X SYBR Green qPCR Master Mix (SeqMatic, Fremont, CA, USA), $1 \mu \mathrm{L}$ of $10 \mu \mathrm{M}$ of each primer, and $1 \mu \mathrm{L}$ of DNA template. Each sample was denatured at $95{ }^{\circ} \mathrm{C}$ for $10 \mathrm{~min}$ before undergoing 25 cycles of $95{ }^{\circ} \mathrm{C}$ for $15 \mathrm{~s}, 60{ }^{\circ} \mathrm{C}$ for $1 \mathrm{~min}$ and $72{ }^{\circ} \mathrm{C}$ for $1.5 \mathrm{~min}$ and a final extension at $72{ }^{\circ} \mathrm{C}$ for $10 \mathrm{~min}$. The amplicons were pooled, purified with the DNA/RNA Purification Beads (SeqMatic, Fremont, CA, USA), and resuspended in 30 $\mu \mathrm{L}$ of $10 \mathrm{mM}$ Tris, $\mathrm{pH} 8.5$. The final purified library was quantified with the 2200 TapeStation (Agilent, Santa Clara, CA, USA). The Purified Library pool was quantified using Qubit and 2200 Tapestation and then diluted to $20 \mathrm{pM}$. The pool was then denatured at $94{ }^{\circ} \mathrm{C}$ on the MJ Research PTC-200 Thermal Cycler and hybridized to Illumina's HT1 buffer. Final quantification was done using qPCR on the Roche Lightcycler 96 system. Using results from the final qPCR, the pool was loaded into a 300 cycle Illumina MiSeq Cartridge at 5 pM and sequenced to generate 150 paired-end reads. Sequencing was performed via Illumina MiSeq.

For $16 \mathrm{~S}$ rRNA amplicon library preparation and sequencing of colon contents from germ-free mice inoculated with the 14 human Clostridia isolates and 3 human Erysipelotrichia isolates, Primers 319F (TCGTCGGCAGCG TCAGATGTGTATAAGAGA CAG(spacer)GTACTCCTACGGGAG GCA GCAGT ) and 806R (GTCTCGTGGGCTCGGAGATGTGTA TAAGAGACAG(spacer)CCGGACTACNVGGGTWT $C T A A T$ were used to amplify the V3-V4 domain of the $16 \mathrm{~S}$ rRNA using a two-step PCR procedure. In step one of the amplification procedure, both forward and reverse primers contained an Illumina tag sequence (bold), a variable length spacer (no spacer, C, TC, or ATC for 319F; no spacer, G, TG, ATG for 806R) to increase diversity and improve the quality of the sequencing run, a linker sequence (italicized), and the $16 \mathrm{~S}$ target sequence (underlined). Each $25 \mu \mathrm{l}$ PCR reaction contained 1 Unit Kapa2G Robust Hot Start Polymerase (Kapa Biosystems), $1.5 \mathrm{mM} \mathrm{MgCl}_{2}, 0.2 \mathrm{mM}$ final concentration dNTP mix, $0.2 \mu \mathrm{M}$ final concentration of each primer, and $1 \mu \mathrm{l}$ of DNA for each sample. PCR conditions were an initial incubation at $95^{\circ} \mathrm{C}$ for $3 \mathrm{~min}$, followed by 25 cycles of $95{ }^{\circ} \mathrm{C}$ for $45 \mathrm{~s}, 50{ }^{\circ} \mathrm{C}$ for $30 \mathrm{~s}$, $72{ }^{\circ} \mathrm{C}$ for $30 \mathrm{~s}$ and a final extension of $72{ }^{\circ} \mathrm{C}$ for $3 \mathrm{~min}$. In step two, each sample was barcoded with a unique forward and reverse barcode combination using forward primers (AATGATACGGCGACCACCGAGATC TACACNNNNNNNNTCGTCGGCAGCGTC) with an Illumina P5 adapter sequence (bold), a unique $8 \mathrm{nt}$ barcode $(\mathrm{N})$, a partial matching sequence of the forward adapter used in step one (underlined), and reverse primers (CAAGCAGAAGACGGCATACGAGAT NNNNNNNNGTCTCGTGGGCTCGG)) with an Illumina P7 adapter sequence (bold), unique 8 nt barcode $(\mathrm{N})$, and a partial matching sequence of the reverse adapter used in step one (underlined). The PCR reaction in step two contained 1 Unit Kapa2G Robust Hot Start Polymerase (Kapa Biosystems), $1.5 \mathrm{mM} \mathrm{MgCl}_{2}, 0.2 \mathrm{mM}$ final concentration dNTP mix, $0.2 \mu \mathrm{M}$ final concentration of each uniquely barcoded primer, and $1 \mu \mathrm{l}$ of the product from the PCR reaction in step one diluted at a 10:1 ratio in water. PCR conditions were an initial incubation at $95{ }^{\circ} \mathrm{C}$ for $3 \mathrm{~min}$, followed by 8 cycles of $95^{\circ} \mathrm{C}$ for $30 \mathrm{~s}, 58{ }^{\circ} \mathrm{C}$ for $20 \mathrm{~s}, 72{ }^{\circ} \mathrm{C}$ for $20 \mathrm{~s}$, and a final extension of $72{ }^{\circ} \mathrm{C}$ for $3 \mathrm{~min}$.

The final product was quantified on the Qubit instrument using the Qubit Broad Range DNA kit (Invitrogen), and individual amplicons were pooled in equal concentrations. The pooled library was cleaned utilizing Ampure XP beads (Beckman Coulter), then the band of interest was further subjected to isolation via gel electrophoresis on a $1.5 \%$ Blue Pippin HT gel (Sage Science). The library was quantified via qPCR followed by 300-bp paired-end sequencing using an Illumina MiSeq instrument in the Genome Center DNA Technologies Core, University of California, Davis.

\section{S rRNA gene amplicon sequencing analysis}

Sequencing reads were demultiplexed using QIIME 1.8 [43], and non-biological nucleotides were trimmed using Trimmomatic [44]. 16S rRNA sequencing reads were subsequently processed and assembled into amplicon sequence variants (ASV) using dada2 [45] in R. First, reads with more than 2 expected errors were removed. Dereplication and sample inference were then performed on forward and reverse reads, prior to merging. A sequence table was constructed from merged reads, and chimeric reads were subsequently removed. Taxonomy was assigned to reads to the genus level using the dada2 formatted rdp training dataset 14 which can be found here: https://zenodo.org/record/158955\#. XJqlnxNKjUI. The $\mathrm{R}$ package phyloseq [46] was then used in downstream analysis of the data, including the 
generation of a phyloseq object, relative abundance bar plots, and the principle coordinate analysis plot. For the weighted unifrac analysis, the $R$ package msa [47] was used to generate a multiple sequence alignment from the assembled reads with the following parameters: method $=$ "ClustalW", type $=$ "dna", order $=$ "input". The $\mathrm{R}$ package phangorn [48] was used to generate a maximum likelihood tree from the sequence alignment using a general time reversible (GTR) model with the following parameters: model = "GTR", optInv $=$ TRUE, optGamma $=$ TRUE, rearrangement $=$ "stochastic", control $=$ pml.control $($ trace $=0)$. The $R$ package vegan was used to perform permanova analysis on weighted unifrac distances with default parameters and the R package ggplot2 was used to graph boxplots of the weighted unifrac distances. The diversity pie chart was generated by computing the ratio of ASVs within each taxonomic class level present to the total number using the R package plyr and then graphed with ggplot 2 . The relative abundance pie chart was generated by using phyloseq and then graphed using ggplot2. For linear discriminant analysis, data were reformatted in $\mathrm{R}$, written to a tab separated text file, and then uploaded to the LEfSe galaxy server [49] where the default statistical parameters were used in the analysis to generate LDA scores and the LDA cladogram. Differential abundance analysis of taxa was performed using the $\mathrm{R}$ package deseq2 [25516281] with the parameters: test $=$ "Wald", fitType $=$ "parametric", cooksCutoff $=$ FALSE. The $\mathrm{R}$ package omu [50] was then used to generate fold change frequency tables and differential abundance bar plots from the deseq 2 modeled data.

For 16S rRNA sequencing analysis of colon contents from ex-germ-free mice inoculated with the 14 human Clostridia isolates and 3 human Erysipelotrichia isolates, reads were assigned strain level taxonomy by creating a local nucleotide blast database of the corresponding genomes using command line blast and then querying each of the 26 ASVs against the database. ASVs that matched the same strain (due to dada2 identifying multiple $16 \mathrm{~S}$ gene copies from the same taxon as unique ASVs) were aggregated prior to the construction of the abundance boxplot using a combination of phyloseq and ggplot2.

\section{Phylogeny of human Clostridia and Erysipelotrichia isolates}

The genome for strain 4 was omitted from this analysis because the sequence provided in NCBI was flagged as chimeric. Analysis of the chimeric sequence of strain 4 suggested that it was derived from two strains belonging to the same species, Hungatella hathewayi. To determine the taxonomy of the remaining 13 Clostridia strains and 3 Erysipelotrichia strains, we employed a phylogenetic approach. Sixty-three Clostridia and Erysipelotrichia genomes were curated, along with 3 Alicyclobacilus genomes that were included as an outgroup (Supplementary Table 6). Protein alignments of the 66 curated genomes, along with the 16 strains from the defined community of Clostridia and Erysipelotrichia isolates, were generated using clustal omega [51] for the following single copy genes: $30 \mathrm{~S}$ ribosomal protein $\mathrm{S} 3,30$ S ribosomal protein $\mathrm{S} 7,30 \mathrm{~S}$ ribosomal protein S9, 30 S ribosomal protein S10, 30S ribosomal protein S12, 30 S ribosomal protein S13, 30 S ribosomal protein S17, 50 S ribosomal protein L3, 50 S ribosomal protein L14, $50 \mathrm{~S}$ ribosomal protein $\mathrm{L} 15$, and $50 \mathrm{~S}$ ribosomal protein L16. Alignments were then concatenated, providing a total alignment length of 1822 amino acids. We then used RaxML [52], with the PROTGAMMAWWAG rate of heterogeneity model to generate 20 maximum likelihood trees in order to determine the best scoring tree. Non-parametric boostrapping was subsequently performed to determine the confidence of branch placements. One hundred fifty bootstraps were sufficient for the model to reach convergence. Bootstraps were then used to draw bipartitions to the best fit tree determined by the maximum likelihood model. The tree was imported into $\mathrm{R}$ and visualized using the package ggtree [53].

\section{GC-TOF mass spectrometry}

Data were acquired using the following chromatographic parameters, with more details to be found in [54]. Column: Restek corporation Rtx-5Sil MS (30 m length $\times 0.25 \mathrm{~mm}$ internal diameter with $0.25-\mu \mathrm{m}$ film made of $95 \%$ dimethyl $/ 5 \%$ diphenylpolysiloxane). Mobile phase: Helium. Column temperature: $50-330{ }^{\circ} \mathrm{C}$. Flow

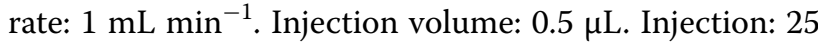
splitless time into a multi-baffled glass liner. Injection temperature: $50{ }^{\circ} \mathrm{C}$ ramped to $250{ }^{\circ} \mathrm{C}$ by $12{ }^{\circ} \mathrm{C} \mathrm{s}^{-1}$. Oven temperature program: $50{ }^{\circ} \mathrm{C}$ for $1 \mathrm{~min}$, then ramped at $20{ }^{\circ} \mathrm{C} \mathrm{min}{ }^{-1}$ to $330{ }^{\circ} \mathrm{C}$, held constant for $5 \mathrm{~min}$. The analytical GC column is protected by a 10-m long empty guard column which is cut by $20-\mathrm{cm}$ intervals whenever the reference mixture $\mathrm{QC}$ samples indicate problems caused by column contaminations. We have validated that at this sequence of column cuts, no detrimental effects are detected with respect to peak shapes, absolute or relative metabolite retention times or reproducibility of quantifications. We use automatic liner exchanges after each set of 10 injections which we could show to reduce sample carryover for highly lipophilic compounds such as free fatty acids. Mass spectrometry parameters are used as follows: a Leco Pegasus IV mass spectrometer is used with unit mass resolution at $17{\text { spectra } \mathrm{s}^{-1}}^{-1}$ 
from $80-500 \mathrm{Da}$ at $-70 \mathrm{eV}$ ionization energy and $1800 \mathrm{~V}$ detector voltage with a $230{ }^{\circ} \mathrm{C}$ transfer line and a $250{ }^{\circ} \mathrm{C}$ ion source.

\section{Metabolomics data processing}

Raw data files are preprocessed directly after data acquisition and stored as ChromaTOF-specific ".peg files, as generic ".txt result files and additionally as generic ANDI MS *.cdf files. ChromaTOF vs. 2.32 is used for data preprocessing without smoothing, $3 \mathrm{~s}$ peak width, baseline subtraction just above the noise level, and automatic mass spectral deconvolution and peak detection at signal/noise levels of 5:1 throughout the chromatogram. Apex masses are reported for use in the BinBase algorithm. Result *.txt files are exported to a data server with absolute spectra intensities and further processed by a filtering algorithm implemented in the metabolomics BinBase database. The BinBase algorithm ( $\mathrm{rtx} 5$ ) used the settings: validity of chromatogram $\left(<10\right.$ peaks with intensity $>10^{7}$ counts $\mathrm{s}^{-1}$ ), unbiased retention index marker detection (MS similarity $>800$, validity of intensity range for high $\mathrm{m} / \mathrm{z}$ marker ions), and retention index calculation by 5 th order polynomial regression. Spectra are cut to 5\% base peak abundance and matched to database entries from most to least abundant spectra using the following matching filters: retention index window \pm 2000 units (equivalent to about $\pm 2 \mathrm{~s}$ retention time), validation of unique ions and apex masses (unique ion must be included in apexing masses and present at $>3 \%$ of base peak abundance), mass spectrum similarity must fit criteria dependent on peak purity and signal/noise ratios and a final isomer filter. Failed spectra are automatically entered as new database entries if $\mathrm{s} / \mathrm{n}>25$, purity $<1.0$, and presence in the biological study design class was $>80 \%$. All thresholds reflect settings for ChromaTOF v. 2.32. Quantification is reported as peak height using the unique ion as default, unless a different quantification ion is manually set in the BinBase administration software BinView. A quantification report table is produced for all database entries that are positively detected in more than $10 \%$ of the samples of a study design class (as defined in the miniX database) for unidentified metabolites. A subsequent post-processing module is employed to automatically replace missing values from the ".cdf files. Replaced values are labeled as 'low confidence' by color coding, and for each metabolite, the number of high-confidence peak detections is recorded as well as the ratio of the average height of replaced values to high-confidence peak detections. These ratios and numbers are used for manual curation of automatic report data sets to data sets released for submission. These data were then normalized to the mTIC value (sum of the peak heights of the known metabolites).

\section{Metabolomics data analysis}

Metabolomics data were loaded into $\mathrm{R}$, and the following analysis was performed using the $\mathrm{R}$ package omu [50]. Compound hierarchy was assigned from the KEGG [55] hierarchy databases in omu's system data. The data were normalized using the natural log, Student's $t$ test was performed to calculate $p$ values, a Benjamini-Hochberg procedure was used to perform false discovery rate correction for type 2 errors, and $\log 2$ fold change of group means was calculated to visualize effect size. Fold change frequency tables were generated to make bar plots. Volcano plots were generated using FDR corrected $p$ values and $\log 2$ fold change between group means. Principle component analysis was performed on natural log transformed data.

\section{D-sorbitol measurement}

Mouse cecal contents were homogenized in $1 \mathrm{~mL}$ of sterile PBS, centrifuged at $300 \mathrm{~g}$ for $10 \mathrm{~min}$ at $4{ }^{\circ} \mathrm{C}$, and then the supernatants were collected. The supernatants were filtered through a $10-\mathrm{kDa}$ spin column (Biovision, Milpitas, CA). D-Sorbitol concentrations were determined by D-sorbitol assay kit (Biovision, Milpitas, CA) according to the manufacturer's instruction.

\section{Supplementary Information}

The online version contains supplementary material available at https://doi. org/10.1186/s40168-021-01123-9.

\section{Additional file 1.}

Additional file 2.

Additional file 3: Figure S1. Amplicon sequence variants (ASVs) belonging to Erysipelotrichia which significantly changed abundance after antibiotic treatment (related to Fig. 2). Mice were mock-treated ( $n=8$ ) or received a single dose of streptomycin $(n=8)$ and bacterial communities in the colon contents were analyzed 3 days later. Box plots showing the relative abundance of Erysipelotrichia ASVs in mock-treated and streptomycin-treated mice.

Additional file 4.

Additional file 5.

Additional file 6.

Additional file 7.

\section{Acknowledgements}

We would like to acknowledge the mass spectrometry-based Metabolomics Service Core at the West Coast Metabolomics Center for expert sample analysis. We would like to acknowledge Briana Young for expert technical assistance. 


\section{Authors' contributions}

CRT analyzed the metabolomics data and microbiota profiling data and wrote the manuscript. AWLR performed and analyzed in vitro growth experiments. EEO helped perform mouse experiments. FF performed mouse experiments and analyzed the data. AJB was a major contributor in writing the manuscript. All authors read and approved the final manuscript.

\section{Funding}

Work in A.J.B.'s laboratory was supported by USDA/NIFA award 2015-6701522930 (A.J.B.), by Crohn's and Colitis Foundation of America Senior Investigator Award \# 650976 and by Public Health Service Grants Al044170, Al096528, Al112445, Al1 12949, Al146432, and Al153069. A.W.L.R. was supported by Public Health Service Grant Al060555. E.E.O. was supported by Public Health Service Grants TR001860 and TR001861.

\section{Availability of data and materials}

Illumina sequences obtained in the present study were deposited in the Sequence Read Archives (SRA) NCBI database under BioProject ID: PRJNA560082. Untargeted metabolomics data are provided in Supplementary Tables 1, 3 and 4 .

\section{Declarations}

\section{Ethics approval and consent to participate}

All experiments in this study were approved by the Institutional Animal Care and Use Committee at the University of California at Davis.

\section{Consent for publication}

Not applicable.

\section{Competing interests}

The authors declare that they have no competing interests.

\section{Author details}

${ }^{1}$ Department of Medical Microbiology and Immunology, School of Medicine, University of California, Davis, One Shields Avenue, Davis, CA 95616, USA. ${ }^{2}$ Present Address: Department of Biological Sciences, California State University Sacramento, 6000 J Street, Sacramento, CA 95819, USA. ${ }^{3}$ Department of Pathology, Microbiology and Immunology, School of Veterinary Medicine, University of California, Davis, One Shields Avenue, Davis, CA 95616, USA. ${ }^{4}$ Present Address: Institute for Molecular Infection Biology (IMIB), Faculty of Medicine, University of Würzburg, Josef-Schneider-Street 2/D15, 97080 Würzburg, Germany.

Received: 12 April 2021 Accepted: 25 June 2021

Published online: 19 August 2021

\section{References}

1. Byndloss MX, Baumler AJ. The germ-organ theory of non-communicable diseases. Nat Rev Microbiol. 2018;16(2):103-10. https://doi.org/10.1038/ nrmicro.2017.158.

2. Martiny AC, Treseder K, Pusch G. Phylogenetic conservatism of functional traits in microorganisms. ISME J. 2013;7(4):830-8. https://doi.org/10.1038/ ismej.2012.160.

3. Doolittle WF. Phylogenetic classification and the universal tree. Science. 1999;284(5423):2124-9.

4. Snel B, Bork P, Huynen MA. Genomes in flux: the evolution of archaeal and proteobacterial gene content. Genome Res. 2002;12(1):17-25. https://doi. org/10.1101/gr.176501.

5. Andreu VP, Augustijn HE, Chen L, Zhernakova A, Fu J, Fischbach MA, et al. A systematic analysis of metabolic pathways in the human gut microbiota. bioRxiv. 2021:2021.02.25.432841. https://doi.org/10.1101/2021.02 25.432841.

6. Rivera-Chavez F, Baumler AJ. The pyromaniac inside you: Salmonella metabolism in the host gut. Annu Rev Microbiol. 2015;69:31-48. https:// doi.org/10.1146/annurev-micro-091014-104108.
7. Rivera-Chavez F, Lopez CA, Baumler AJ. Oxygen as a driver of gut dysbiosis. Free Radic Biol Med. 2017;105:93-101. https://doi.org/10.1016/j.freer adbiomed.2016.09.022.

8. Desai MS, Seekatz AM, Koropatkin NM, Kamada N, Hickey CA, Wolter $M$, et al. A dietary fiber-deprived gut microbiota degrades the colonic mucus barrier and enhances pathogen susceptibility. Cell. 2016;167(5):1339-53.e21. https://doi.org/10.1016/j.cell.2016.10.043.

9. Tasse L, Bercovici J, Pizzut-Serin S, Robe P, Tap J, Klopp C, et al. Functional metagenomics to mine the human gut microbiome for dietary fiber catabolic enzymes. Genome Res. 2010;20(11):1605-12. https://doi.org/10. 1101/gr.108332.110.

10. Jacobson A, Lam L, Rajendram M, Tamburini F, Honeycutt J, Pham T, et al. A gut commensal-produced metabolite mediates colonization resistance to Salmonella infection. Cell Host Microbe. 2018;24(2):296-307.e7. https:// doi.org/10.1016/j.chom.2018.07.002.

11. Flint HJ, Scott KP, Duncan SH, Louis P, Forano E. Microbial degradation of complex carbohydrates in the gut. Gut Microbes. 2012;3(4):289-306. https://doi.org/10.4161/gmic.19897.

12. Wu GD, Chen J, Hoffmann C, Bittinger K, Chen YY, Keilbaugh SA, et al. Linking long-term dietary patterns with gut microbial enterotypes. Science. 2011;334(6052):105-8. https://doi.org/10.1126/science.1208344.

13. Pryde SE, Duncan SH, Hold GL, Stewart CS, Flint HJ. The microbiology of butyrate formation in the human colon. FEMS Microbiol Lett. 2002;217(2):133-9. https://doi.org/10.1111/j.1574-6968.2002.tb11467.x.

14. Vital M, Howe AC, Tiedje JM. Revealing the bacterial butyrate synthesis pathways by analyzing (meta)genomic data. MBio. 2014;5(2):e00889. https://doi.org/10.1128/mBio.00889-14.

15. Donohoe DR, Garge N, Zhang X, Sun W, O'Connell TM, Bunger MK, et al. The microbiome and butyrate regulate energy metabolism and autophagy in the mammalian colon. Cell Metab. 2011;13(5):517-26. https://doi.org/10.1016/j.cmet.2011.02.018.

16. Velazquez OC, Lederer HM, Rombeau JL. Butyrate and the colonocyte. Production, absorption, metabolism, and therapeutic implications. Adv Exp Med Biol. 1997:427:123-34.

17. Zheng L, Kelly CJ, Colgan SP. Physiologic hypoxia and oxygen homeostasis in the healthy intestine. A review in the theme: cellular responses to hypoxia. Am J Physiol Cell Physiol. 2015;309(6):C350-60. https://doi.org/ 10.1152/ajpcell.00191.2015.

18. Byndloss MX, Olsan EE, Rivera-Chávez F, Tiffany CR, Cevallos SA, Lokken $\mathrm{KL}$, et al. Microbiota-activated PPAR-g signaling inhibits dysbiotic Enterobacteriaceae expansion. Science. 2017;357:570-5.

19. Eckburg PB, Bik EM, Bernstein CN, Purdom E, Dethlefsen L, Sargent $\mathrm{M}$, et al. Diversity of the human intestinal microbial flora. Science. 2005;308(5728):1635-8. https://doi.org/10.1126/science.1110591.

20. Ludwig W, Schleifer KH, Whitman WB. Revised road map to the phylum Firmicutes. In: De Vos P, Garrity G, Jones D, Krieg NR, Ludwig W, Rainey FA, Schleifer K-H, Whitman WB, editors. Bergey's manual of systematic bacteriology. New York: Springer; 2009. p. 1-13.

21. Atarashi K, Tanoue T, Shima T, Imaoka A, Kuwahara T, Momose Y, et al. Induction of colonic regulatory $T$ cells by indigenous Clostridium species. Science. 2011;331(6015):337-41. https://doi.org/10.1126/science. 1198469.

22. Antunes LC, Han J, Ferreira RB, Lolic P, Borchers CH, Finlay BB. Effect of antibiotic treatment on the intestinal metabolome. Antimicrob Agents Chemother. 2011;55(4):1494-503. https://doi.org/10.1128/AAC.01664-10.

23. Rivera-Chavez F, Zhang LF, Faber F, Lopez CA, Byndloss MX, Olsan EE, et al. Depletion of butyrate-producing Clostridia from the gut microbiota drives an aerobic luminal expansion of Salmonella. Cell Host Microbe. 2016;19(4):443-54. https://doi.org/10.1016/j.chom.2016.03.004.

24. Gillis CC, Hughes ER, Spiga L, Winter MG, Zhu W, de Carvalho TF, et al. Dysbiosis-associated change in host metabolism generates lactate to support Salmonella growth. Cell Host Microbe. 2018;23(1):54-64.e6. https://doi.org/10.1016/j.chom.2017.11.006.

25. Atarashi K, Tanoue T, Oshima K, Suda W, Nagano Y, Nishikawa H, et al. Treg induction by a rationally selected mixture of Clostridia strains from the human microbiota. Nature. 2013;500(7461):232-6. https://doi.org/10. 1038/nature12331.

26. Lang JM, Darling AE, Eisen JA. Phylogeny of bacterial and archaeal genomes using conserved genes: supertrees and supermatrices. PLoS One. 2013;8(4):e62510. https://doi.org/10.1371/journal.pone.0062510. 
27. Kim YG, Sakamoto K, Seo SU, Pickard JM, Gillilland MG 3rd, Pudlo NA, et al. Neonatal acquisition of Clostridia species protects against colonization by bacterial pathogens. Science. 2017;356(6335):315-9. https://doi.org/ 10.1126/science.aag2029.

28. Furusawa Y, Obata Y, Fukuda S, Endo TA, Nakato G, Takahashi D, et al. Commensal microbe-derived butyrate induces the differentiation of colonic regulatory T cells. Nature. 2013;504(7480):446-50. https://doi.org/10. 1038/nature12721.

29. Belloir C, Neiers F, Briand L. Sweeteners and sweetness enhancers. Curr Opin Clin Nutr Metab Care. 2017;20(4):279-85. https://doi.org/10.1097/ MCO.0000000000000377.

30. Gibson PR, Shepherd SJ. Personal view: food for thought-western lifestyle and susceptibility to Crohn's disease. The FODMAP hypothesis. Aliment Pharmacol Ther. 2005;21(12):1399-409. https://doi.org/10.1111/j.13652036.2005.02506.x.

31. Beaugerie L, Flourie B, Marteau P, Pellier P, Franchisseur C, Rambaud JC. Digestion and absorption in the human intestine of three sugar alcohols. Gastroenterology. 1990;99(3):717-23.

32. Wang YM, van Eys J. Nutritional significance of fructose and sugar alcohols. Annu Rev Nutr. 1981;1:437-75. https://doi.org/10.1146/annurev.nu. 01.070181 .002253 .

33. Gibson PR, Shepherd SJ. Evidence-based dietary management of functional gastrointestinal symptoms: the FODMAP approach. J Gastroenterol Hepatol. 2010;25(2):252-8. https://doi.org/10.1111/j.1440-1746.2009. 06149.x.

34. Grabitske HA, Slavin JL. Low-digestible carbohydrates in practice. J Am Diet Assoc. 2008;108(10):1677-81. https://doi.org/10.1016/j.jada.2008.07. 010.

35. Ong DK, Mitchell SB, Barrett JS, Shepherd SJ, Irving PM, Biesiekierski JR, et al. Manipulation of dietary short chain carbohydrates alters the pattern of gas production and genesis of symptoms in irritable bowel syndrome. J Gastroenterol Hepatol. 2010;25(8):1366-73. https://doi.org/10.1111/j. 1440-1746.2010.06370.x.

36. Halmos EP, Power VA, Shepherd SJ, Gibson PR, Muir JG. A diet low in FODMAPs reduces symptoms of irritable bowel syndrome. Gastroenterology. 2014;146(1):67-75.e5. https://doi.org/10.1053/j.gastro.2013.09.046.

37. Bartlett JG, Moon N, Chang TW, Taylor N, Onderdonk AB. Role of Clostridium difficile in antibiotic-associated pseudomembranous colitis. Gastroenterology. 1978;75(5):778-82.

38. Theriot CM, Koenigsknecht MJ, Carlson PE Jr, Hatton GE, Nelson AM, $\mathrm{Li} B$, et al. Antibiotic-induced shifts in the mouse gut microbiome and metabolome increase susceptibility to Clostridium difficile infection. Nat Commun. 2014;5:3114. https://doi.org/10.1038/ncomms4114.

39. Hofmann JD, Biedendieck R, Michel AM, Schomburg D, Jahn D, Neumann-Schaal M. Influence of L-lactate and low glucose concentrations on the metabolism and the toxin formation of Clostridioides difficile. PLOS One. 2021;16(1):e0244988. https://doi.org/10.1371/journal.pone.0244988.

40. Ghimire S, Roy C, Wongkuna S, Antony L, Maji A, Keena MC, et al. Identification of Clostridioides difficile-inhibiting gut commensals using culturomics, phenotyping, and combinatorial community assembly. mSystems. 2020;5(1). https://doi.org/10.1128/mSystems.00620-19.
41. Oliveira RA, Ng KM, Correia MB, Cabral V, Shi H, Sonnenburg JL, et al. Klebsiella michiganensis transmission enhances resistance to Enterobacteriaceae gut invasion by nutrition competition. Nat Microbiol. 2020;5(4):630-41. https://doi.org/10.1038/s41564-019-0658-4.

42. Narushima S, Sugiura Y, Oshima K, Atarashi K, Hattori M, Suematsu M, et al. Characterization of the 17 strains of regulatory $T$ cell-inducing human-derived Clostridia. Gut Microbes. 2014;5(3):333-9. https://doi.org/ 10.4161/gmic.28572.

43. Caporaso JG, Kuczynski J, Stombaugh J, Bittinger K, Bushman FD, Costello EK, et al. QIIME allows analysis of high-throughput community sequencing data. Nat Methods. 2010;7(5):335-6. https://doi.org/10.1038/nmeth.f. 303.

44. Bolger AM, Lohse M, Usadel B. Trimmomatic: a flexible trimmer for Illumina sequence data. Bioinformatics. 2014;30(15):2114-20. https://doi. org/10.1093/bioinformatics/btu170

45. Callahan BJ, McMurdie PJ, Rosen MJ, Han AW, Johnson AJ, Holmes SP. DADA2: high-resolution sample inference from Illumina amplicon data. Nat Methods. 2016;13(7):581-3. https://doi.org/10.1038/nmeth.3869.

46. McMurdie PJ, Holmes S. phyloseq: an R package for reproducible interactive analysis and graphics of microbiome census data. PLoS One. 2013;8(4):e61217. https://doi.org/10.1371/journal.pone.0061217.

47. Bodenhofer U, Bonatesta E, Horejs-Kainrath C, Hochreiter S. msa: an $\mathrm{R}$ package for multiple sequence alignment. Bioinformatics. 2015;31(24):3997-9. https://doi.org/10.1093/bioinformatics/btv494.

48. Schliep KP. phangorn: phylogenetic analysis in R. Bioinformatics. 2011;27(4):592-3. https://doi.org/10.1093/bioinformatics/btq706.

49. Segata N, Izard J, Waldron L, Gevers D, Miropolsky L, Garrett WS, et al. Metagenomic biomarker discovery and explanation. Genome Biol. 2011;12(6):R60. https://doi.org/10.1186/gb-2011-12-6-r60.

50. Tiffany CR, Baumler AJ. omu, a metabolomics count data analysis tool for intuitive figures and convenient metadata collection. Microbiol Resour Announc. 2019;8(15). https://doi.org/10.1128/MRA.00129-19.

51. Sievers F, Higgins DG. Clustal Omega, accurate alignment of very large numbers of sequences. Methods Mol Biol. 2014;1079:105-16. https://doi. org/10.1007/978-1-62703-646-7_6.

52. Stamatakis A. RAxML version 8: a tool for phylogenetic analysis and postanalysis of large phylogenies. Bioinformatics. 2014;30(9):1312-3. https:// doi.org/10.1093/bioinformatics/btu033.

53. Yu G. Using ggtree to visualize data on tree-like structures. Curr Protoc Bioinformatics. 2020;69(1):e96. https://doi.org/10.1002/cpbi.96.

54. Fiehn O, Wohlgemuth G, Scholz M, Kind T, Lee DY, LuY, et al. Quality control for plant metabolomics: reporting MSI-compliant studies. Plant J. 2008;53(4):691-704. https://doi.org/10.1111/j.1365-313X.2007.03387.x.

55. Kanehisa M, Goto S. KEGG: kyoto encyclopedia of genes and genomes. Nucleic Acids Res. 2000;28(1):27-30. https://doi.org/10.1093/nar/28.1.27.

\section{Publisher's Note}

Springer Nature remains neutral with regard to jurisdictional claims in published maps and institutional affiliations.

\footnotetext{
Ready to submit your research? Choose BMC and benefit from:

- fast, convenient online submission

- thorough peer review by experienced researchers in your field

- rapid publication on acceptance

- support for research data, including large and complex data types

- gold Open Access which fosters wider collaboration and increased citations

- maximum visibility for your research: over 100M website views per year
}

At $\mathrm{BMC}$, research is always in progress.

Learn more biomedcentral.com/submissions 